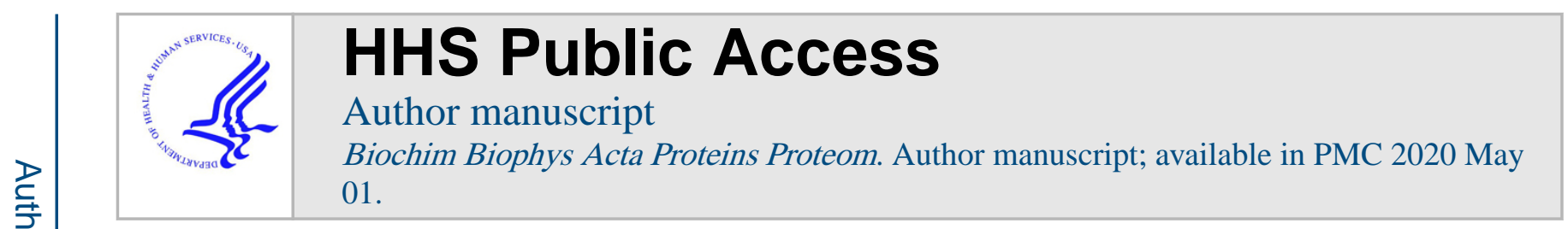

Published in final edited form as:

Biochim Biophys Acta Proteins Proteom. 2019 May ; 1867(5): 529-536. doi:10.1016/j.bbapap. 2018.11.006.

\title{
Zinc Boosts EGCG's hIAPP Amyloid Inhibition Both in Solution and Membrane
}

\author{
Young-Ho Lee ${ }^{\# a, b}$, Yuxi Lin ${ }^{\# c}$, Sarah J. Cox ${ }^{d}$, Misaki Kinoshitaa ${ }^{a}$ Bikash R. Sahood, \\ Magdalena Ivanova ${ }^{\mathrm{e}}$, and Ayyalusamy Ramamoorthy ${ }^{\mathrm{d}}$ \\ a Institute for Protein research, Osaka University Yamadaoka 3-2, Suita, Osaka 565-0871, Japan. \\ b Protein Structure Research Group, Division of Bioconvergence Analysis, Korea Basic Science \\ Institute, Chungcheongbuk-do 28119, South Korea. \\ c Department of Chemistry, Sookmyung Women's University Cheongpa-ro 47-gil 100, Yongsangu, \\ Seoul 04310, South Korea. \\ d Biophysics and Department of Chemistry, University of Michigan Ann Arbor, MI 48109-1055 \\ e Department of Neurology, University of Michigan, Ann Arbor, MI 48109, USA \\ \# These authors contributed equally to this work.
}

\begin{abstract}
Amyloid aggregation of human islet amyloid polypeptide (hIAPP) is linked to insulin-producing islet cell death in type II diabetes. Previous studies have shown that Zinc (Zn(II)) and insulin, cosecreted with hIAPP, have an inhibition effect on hIAPP aggregation. Lipid membranes have also been shown to significantly influence the aggregation kinetics of hIAPP. An increasing number of studies report the importance of developing small molecule inhibitors to suppress the hIAPP's aggregation and subsequent toxicity. The ability of epigallocatechin-gallate (EGCG) to inhibit aggregation of a variety of amyloid peptide/proteins initiated numerous studies as well as the development of derivative compounds to potentially treat amyloid diseases. In this study, a combination of Thioflavin- T fluorescence kinetics, transmission electron microscopy, isothermal titration calorimetery, circular dicrosim and nucelar magnetic resonance experiments were used to demonstrate a significant enhancement in EGCG's efficiency when complexed with $\mathrm{Zn}$ (II). We demonstrate that the Zn-EGCG complex is able to significantly suppress hIAPP's amyloid aggregation both in presence and absence of lipid membrane. Circular dichroism experiments indicate the formation and stabilization of a helical structure of hIAPP in presence of the EGCG:Zn(II) complex. Our results also reveal the ability of EGCG or EGCG:Zn(II) to efficiently suppress hIAPP's cellular toxicity. We believe that the reported results could be useful to develop strategies to trap hIAPP intermediates for further biophysical and structural studies, and also to devise approaches to abolish amyloid aggregation and cellular toxicity.
\end{abstract}

\section{Graphical Abstract}

Publisher's Disclaimer: This is a PDF file of an unedited manuscript that has been accepted for publication. As a service to our customers we are providing this early version of the manuscript. The manuscript will undergo copyediting, typesetting, and review of the resulting proof before it is published in its final citable form. Please note that during the production process errors may be discovered which could affect the content, and all legal disclaimers that apply to the journal pertain. 


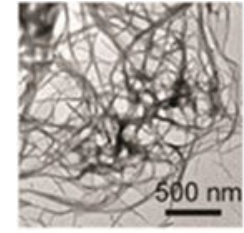

hIAPP+LUVs

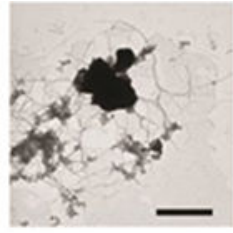

hIAPP+LUVs + EGCG

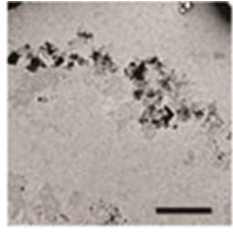

hIAPP+LUVs

$+\mathrm{EGCG}+\mathrm{Zn}(\mathrm{II})$

\section{Keywords}

epigallocatechin-gallate; islet amyloid polypeptide; type II diabetes; amyloid; membrane; protein aggregation

\section{Introduction}

Human-IAPP (hIAPP: human islet amyloid polypeptide, also known as amylin) is a 37 residue peptide hormone (Fig. S1A) that is co-secreted at a 1:100 molar ratio with insulin in response to blood glucose levels that normally contributes to satiety and the slowing of gastric emptying.[1] In type II diabetes, the increase in insulin production causes a simultaneous increase in the generation of hIAPP, which increases hIAPP amyloid formation resulting in $\beta$ cell death.[2,3] Biophysical studies have reported that hIAPP aggregation results in the disruption of lipid membranes via a two-step mechanism: fibrilindependent pore formation and a fibril-dependent fragmentation of the lipid bilayer.[4] The presence of anionic lipids enhances monomeric hIAPP binding to the lipid membrane while the membrane curvature plays a role in the rate of aggregation. [5] Previous studies have also reported that hIAPP self-assembles to form helical oligomers.[6-8]

Due to the cellular toxicity that is associated with amyloid formation, it is important to develop molecules that inhibit aggregation and toxicity.[9-11] One of the most frequently investigated amyloid inhibitors is epigallocatechin-gallate (EGCG) (Fig. S1B), a polyphenolic compound found in green tea extract.[12-16] EGCG has been shown to be an effective aggregation inhibitor for a variety of amyloid-forming peptides and proteins.[1721] Metal ions have also been shown to play a role in many types of amyloid aggregation. Zinc is highly abundant in pancreatic $\beta$ cells and Zinc deficiency is a common symptom in type II diabetes.[22] Zinc has been shown to coordinate with His-18 of hIAPP and promotes the formation of off-pathway oligomers that are incompetent to form amyloid fibers.

Interestingly, Zinc has a dual effect on hIAPP's aggregation in which low concentrations of Zinc inhibit fiber formation but high concentrations promote aggregation.[23-25] Since hIAPP aggregation induced membrane disruption is linked to islet cell death in type II diabetes, it is important to examine the efficiency of amyloid inhibition by small molecules in a membrane environment. In this study, we examined the combined effects of EGCG and Zinc (EGCG:Zn(II)) as a complex on hIAPP's aggregation, structure, and toxicity in the presence and absence of lipid membranes. Our results show that Zinc enhances the ability of EGCG to inhibit amyloid formation. By using a combination of Thioflavin-T (ThT) based fluorescence and transmission electron microscopy (TEM) experiments, we show significant 
increase in the lag-time, reduction in the elongation rate, and amorphous aggregate formation in the presence of EGCG or EGCG:Zn(II) complex. It is remarkable that EGCG:Zn(II) stabilizes a helical structure of hIAPP in a lipid membrane environment. Our results also show that EGCG:Zn(II) complex also increases the cell viability by suppressing zinc's toxicity in cells.

\section{Materials and Methods}

\subsection{Chemicals}

Lyophilized hIAPP with amidated C-termini was purchased from Peptide Institute Inc (Osaka, Japan). In order to avoid undesired aggregation, hIAPP solution was carefully prepared following a previously published procedure.[26] The concentration of hIAPP was spectroscopically determined based on a molar extinction coefficient of $1490 \mathrm{M}^{-1} \mathrm{~cm}^{-1}$ at $280 \mathrm{~nm}$. 1-palmitoyl-2oleoyl-sn-glycero-3-phosphocholine (POPC) and 1-palmitoyl-2oleoyl-sn-glycero-3-phospho-rac(1-glycerol) (POPG) were purchased from Avanti Polar Lipids, Inc. (Alabaster, AL). ThT was obtained from Wako Pure Chemical Industries, Ltd. $\mathrm{ZnCl}_{2}$, and EGCG and the other reagents were purchased from Nacalai Tesque (Kyoto, Japan).

\subsection{Preparation of lipid vesicles}

Large unilamellar vesicles (LUVs) with a diameter of $100 \mathrm{~nm}$ were prepared as described previously.[27] Briefly, POPC and POPG lipids were first dissolved in chloroform and mixed at a 7:3 POPC:POPG molar ratio for mimicking a mixed anionic/zwitterionic membrane system. The solvent was removed under a steam of nitrogen gas and dried overnight to generate a lipid film which was then rehydrated in $20 \mathrm{mM}$ HEPES (pH 7.4) containing $50 \mathrm{mM} \mathrm{NaCl}$. Lipid suspension was subjected to 5 rounds of freeze-thaw and the resulting solution was extruded 23 times through 100 -nm polycarbonate membranes (Whatman, Clifton, NJ) using a mini-extruder (Avanti Polar Lipids, Inc., Alabaster, AL). Unless otherwise noted, LUVs consisting of $250 \mu \mathrm{M}$ lipids were used in this study.

\subsection{ThT assay for amyloid formation of hIAPP}

Kinetics of amyloid fibrillation at $37^{\circ} \mathrm{C}$ was monitored using $5 \mu \mathrm{M}$ hIAPP in $20 \mathrm{mM}$ HEPES buffer (pH 7.4) containing $5 \mu \mathrm{M}$ ThT and $50 \mathrm{mM} \mathrm{NaCl}$ in the presence and absence of LUVs. Other effectors of hIAPP amyloid aggregation including Zn(II), EGCG, or 1:1 molar ratio of EGCG:Zn(II) were added to the hIAPP sample solution at the desired concentration. Three independent experimental measurements were carried out for each sample.

ThT-based monitoring was performed using a sealed 96-well microplate (Greiner-Bio-One, Tokyo, Japan). ThT fluorescence intensity was observed in a microplate reader (MTP-810, Corona Electric Co. Ibaraki, Japan) every 3 min following a $10 \mathrm{~s}$ orbital shaking (strong mode). The excitation and emission wavelengths were set at 450 and $490 \mathrm{~nm}$, respectively. Kinetic parameters of amyloid formation were obtained using the following equation;[28] 


$$
\mathrm{Y}=y_{i}+m_{i} \mathrm{t}+\frac{y_{f}+m_{f} t}{1+\exp \left[-k\left(t-t_{0}\right)\right]}
$$

where $y_{\mathrm{i}}+m_{\mathrm{i}} t$ and $y_{\mathrm{f}}+m_{\mathrm{f}} t$ indicate initial and final baselines, respectively. $k$ and $t$ indicate the rate constant and elongation time, respectively. $t_{0}$ is the half-time when ThT fluorescence reaches $50 \%$ of the maximum intensity. The lag time was calculated with the relationship, lag time $=t_{0}-2(1 / k)$.

\subsection{Circular dichroism spectroscopy}

Far-UV circular dichroism (CD) spectra were obtained from a sample of $15 \mu \mathrm{M}$ hIAPP dissolved in $20 \mathrm{mM}$ HEPES containing $50 \mathrm{mM} \mathrm{NaCl}$ and $500 \mu \mathrm{M}$ lipid in LUVs. Zn(II), EGCG, or EGCG:Zn(II) (1:1) complex was added to the hIAPP sample solution at the desired concentration. CD spectra were also obtained in the absence of LUVs to measure conformational changes in hIAPP $(15 \mu \mathrm{M})$ in presence of two equivalents of EGCG or EGCG:Zn(II). CD measurements were performed on a Jasco J820 spectropolarimeter (Tokyo, Japan) using a quartz cuvette with a light path of $0.1 \mathrm{~cm}$. A cell holder equipped with a water circulator was used to keep the sample temperature at $37^{\circ} \mathrm{C}$. Spectra were presented as mean residue ellipticity, $[\theta]\left(\mathrm{deg} \mathrm{cm}^{2} \mathrm{dmol}^{-1}\right)$, after subtracting the CD spectrum of the solvent without hIAPP. CD spectra of hIAPP interacting with LUVs in the absence (after $45 \mathrm{~min}$ incubation) and presence of $\mathrm{Zn}(\mathrm{II})$ (after $45 \mathrm{~min}$ incubation), EGCG (after 90 min incubation), and 1:1 EGCG:Zn(II) (after 90 min incubation) were obtained for secondary structural analysis.

\subsection{Transmission electron microscopy}

All TEM images were obtained using a HITACHI H-7650 transmission microscope (Hitachi, Tokyo, Japan) at $20^{\circ} \mathrm{C}$ with a voltage of $80 \mathrm{kV}$. Each sample solution was applied onto a collodion-coated copper grid (Nisshin EM Co., Tokyo, Japan), and negatively stained with $2 \%$ (weight/weight) ammonium molybdate as reported previously.[27]

\subsection{Preparation of EGCG:Zn(II) complex}

EGCG: $\mathrm{Zn}(\mathrm{II})$ complex was prepared by mixing $\mathrm{ZnCl}_{2}$ and EGCG at the desired molar ratio in $20 \mathrm{mM}$ HEPES buffer ( $\mathrm{pH} 7.4$ ) containing $50 \mathrm{mM} \mathrm{NaCl}$. The absorption spectra of EGCG:Zn(II) complex were acquired using Hitachi U-3000 spectrometer (Hitachi, Tokyo, Japan).

\subsection{Cell toxicity experiments}

To prepare hIAPP fibrils for the cell proliferation assays, lyophilized hIAPP monomer was solubilized in Milli-Q water which was adjusted to $\mathrm{pH} 4 \mathrm{using} \mathrm{HCl}$. Stock solutions were prepared by adding sample buffer ( $50 \mathrm{mM} \mathrm{NaCl}, 20 \mathrm{mM}$ HEPES, $\mathrm{pH} 7.4$ ) to a final concentration of $0.1 \mathrm{mg} / \mathrm{ml}$. Peptide solutions at $100 \mu \mathrm{M}$ were then treated with either 1 or 5 molar equivalents of EGCG:Zn(II). To prepare hIAPP aggregates, peptides were incubated at room temperature with shaking at $750 \mathrm{rpm}$ for 24 hours. RIN-5F cells (purchased from ATCC, cat\# CRL-2058, 61465080) were grown in RPMI-1640 media with 2 mM L- 
glutamine supplemented with $10 \%$ fetal bovine serum in a humidified incubator at $37{ }^{\circ} \mathrm{C}$ with $5 \% \mathrm{CO}_{2}$. Cells were kept between passage numbers 5-15. Cells were cultured in 10-cm cell culture dishes. For cell viability assays, $90 \mu \mathrm{l}$ of cells were dispensed to a total of 30,000 cells per well in 96 flat-bottom well trays and incubated for 24 hours at $37^{\circ} \mathrm{C}$ with $5 \% \mathrm{CO}_{2}$ prior to the assay. Cell proliferation was measured by 3-(4,5-dimethylthiazol2-yl)-2,5-diphenyltetrazolium bromide (MTT) assay (CellTiter 96 Non-Radioactive Cell Proliferation Assay, Promega). $10 \mu \mathrm{L}$ of peptides (freshly dissolved and aggregated) were added to each well to a final concentration of 1,5 or $10 \mu \mathrm{M}$. After $24 \mathrm{~h}$ incubation the cell proliferation was determined as described by the manufacturer. In short, $15 \mu \mathrm{L}$ dye solution was added to each well. After incubation at $37{ }^{\circ} \mathrm{C}$ for 3-3.5 hours, $100 \mu \mathrm{L}$ of solubilization solution/stop mix was added. Plates were left at room temperature overnight with gentle shaking. The absorbance was measured at 570 and $700 \mathrm{~nm}$ (background). Data was corrected for the background by subtracting the absorbance at $700 \mathrm{~nm}$ from the absorbance at $570 \mathrm{~nm}$. The data was normalized with cells treated with $1 \%(\mathrm{w} / \mathrm{v})$ SDS to $0 \%$ reduction, and cells treated with a sample buffer to $100 \%$ reduction. Five technical replicates were used for each experiment and the error reported is the standard deviation of these experiments. Each experiment was repeated three times. Ordinary one-way ANOVA tests with Tukey's multiple comparisons were performed in respect to non-treated cells (dotted line) and individual samples (solid line). ****p $<0.0001, * * * \mathrm{p}<0.0002, * * \mathrm{p}<0.01$ and $* \mathrm{p}<0.05$ indicate levels of significant differences.

\subsection{Dynamic light scattering measurements}

EGCG solutions were prepared in $20 \mathrm{mM}$ HEPES buffer (pH 7.4) containing $50 \mathrm{mM} \mathrm{NaCl}$ and mixed well by gently vortexing for $30 \mathrm{~s}$. A solution mixture of EGCG and $\mathrm{ZnCl}_{2}$ was prepared at equimolar concentration and incubated for 15 minutes at $37^{\circ} \mathrm{C}$. The size distribution of EGCG or EGCG:Zn(II) complex solutions were measured by dynamic light scattering (DLS) at $37^{\circ} \mathrm{C}$. All DLS experiments were performed using a DynaPro NanoStar from Wyatt Technology (Santa Barbara, CA) with a 1- $\mu \mathrm{L}$ quartz cuvette and the average values over 20 independent scans are presented.

\subsection{Nuclear magnetic resonance spectroscopy}

Proton NMR spectra of hIAPP were recorded on a $600 \mathrm{MHz}$ Bruker Avance III spectrometer equipped with a z-axis gradient cryogenic probe at $10^{\circ} \mathrm{C}$. Freshly dissolved hIAPP monomers $(50 \mu \mathrm{M})$ in $20 \mathrm{mM}$ HEPES buffer ( $\mathrm{pH} 6.5$ ) containing $50 \mathrm{mM} \mathrm{NaCl}, 90 \% \mathrm{H}_{2} \mathrm{O}$, and $10 \% \mathrm{D}_{2} \mathrm{O}$ were titrated with either $500 \mu \mathrm{M}$ EGCG or $500 \mu \mathrm{M}$ EGCG:Zn(II) mixture. NMR spectra were processed using TopSpin 3.5 (Bruker BioSpin, Germany).

\subsection{Isothermal titration calorimetry}

Isothermal titration calorimetry (ITC) experiments were performed at $37{ }^{\circ} \mathrm{C}$ with a VP-ITC instrument (Malvern Instrument, UK). 1 mM EGCG or EGCG:Zn(II) (1:1 molar ratio) in the ITC syringe was titrated to $60 \mu \mathrm{M}$ hIAPP placed in the ITC cell. A $20 \mathrm{mM}$ HEPES buffer (pH 7.4) containing $50 \mathrm{mM} \mathrm{NaCl}$ was used. Sample solutions were kept at $0{ }^{\circ} \mathrm{C}$ (ice) before the injection. The value of differential power for the baseline was set to $10 \mu \mathrm{cal} \mathrm{s}^{-1}$. Titration experiments consisted of 40 injections spaced at 25 min intervals. The injection volume was 
$2 \mu \mathrm{l}$ over 4 seconds for the first titration and $7 \mu$ over 14 seconds for the remaining titrations. The cell was continuously stirred at $307 \mathrm{rpm}$.

\section{Results and Discussion}

\subsection{Effect of EGCG and Zn(II) on hIAPP aggregation in LUVs}

The formation of EGCG:Zn(II) complex in solution (Fig.S2) and in the presence of POPC:POPG(7:3 molar ratio) LUVs (Fig.S3) was first characterized using UV-Vis experiments. Experimental results reveal that EGCG is capable of binding to more than one unit of $\mathrm{Zn}$ (II) in solution and that the formation of EGCG:Zn(II) complex is possible even in the membrane environment, which is in agreement with results previously reported.[29] DLS experiments provided information on physical phase states of EGCG and EGCG:Zn(II) (Fig. S4). The hydrodynamic radius $\left(R_{\mathrm{H}}\right)$ of EGCG was predominantly distributed around $\sim 7-8 \mathrm{~nm}$ with a polydispersity index (Pd) of $2.5 \times 10^{-2}$ while the $R_{\mathrm{H}}$ of EGCG: $\mathrm{Zn}$ (II) displayed multiple signals around $\sim 5 \mathrm{~nm}\left(\mathrm{Pd}=1.4 \times 10^{-2}\right), \sim 250 \mathrm{~nm}\left(\mathrm{Pd}=3.4 \times 10^{-2}\right)$, and $\sim 300$ $400 \mathrm{~nm}\left(\mathrm{Pd}=2.2 \times 10^{-2}\right)$. This indicates that EGCG alone exists in a small aggregated form while the EGCG:Zn(II) complex forms larger aggregates (Fig. S4).

Since both Zn(II) and EGCG are independently capable of interacting with hIAPP and suppressing its fibril formation, the efficiencies of Zn(II), EGCG, and the EGCG:Zn(II) complex to inhibit aggregation of hIAPP in solution (Fig. S5) and with POPC:POPG LUVs were examined by ThT kinetic experiments. The results shown in Fig. 1 reveal the ability of EGCG or the EGCG:Zn(II) complex to inhibit the fibrillation of hIAPP not only in the absence but also in the presence of lipid bilayers. For a better comparison of the experimentally observed effects of Zn(II), EGCG, and EGCG:Zn(II) complex on the fibril formation of hIAPP, the observed changes in the lag-time, elongation rate constant, and maximum ThT intensity are summarized in Fig. 1(D-F). Zn(II) alone slightly increases the lag-time and slightly reduces the maximum ThT intensity without affecting the elongation rate constant (Fig.1A and D-F). On the other hand, both EGCG and EGCG:Zn(II) mixture exhibited a considerable increase in the lag-time as well as a reduction in both elongation rate constant and maximum ThT intensity (Fig.1B-F). For example, the elongation rate constant and ThT intensity are fully suppressed by 25-50 $\mu \mathrm{M}$ EGCG without an appreciable lag-time (Fig. 1E). The complex of EGCG:Zn(II) enhanced these effects in the presence of membrane and was sufficient to dramatically reduce both the elongation rate and ThT intensity (Fig.1E and F). At $2.5 \mu \mathrm{M}$ of 1:1 EGCG:Zn(II), amyloid formation was completely prevented in solution as indicated by the remarkably low ThT fluorescence intensity (Fig. S5C), while a high ThT fluorescence intensity was still observed with a slow apparent elongation rate and prolonged lag-time in the absence of $\mathrm{Zn}$ (II) (Fig. S5B).

Since EGCG is capable of interfering with ThT's ability to bind to amyloid fibrils as reported elsewhere, $[17,30]$ the observed inhibition of fibril formation was confirmed using TEM (Fig. 2). As shown in the TEM images, $\mathrm{Zn}$ (II) alone is capable of reducing the overall fibril density (Fig. 2B), which is in agreement with a previous study.[24] On the other hand, both EGCG and EGCG:Zn(II) are fairly efficient in fully suppressing the fibril formation by promoting the formation of amorphous aggregates (Fig. 2C-F). It is remarkable that the amount of EGCG required to completely suppress hIAPP fibril formation is dramatically 
reduced by the presence of $\mathrm{Zn}(\mathrm{II})$ as displayed from experimental results shown in Figs. 1 (D-F) and S5 (B and C) and TEM in Fig. 2.

\subsection{EGCG:Zn(II) complex reduces the $\beta$-sheet content of hIAPP aggregates}

The ThT and TEM results were further confirmed by CD experiments (Fig. 3).

Conformational analysis of hIAPP without any of the additives used in this study showed a random-coil like conformation with the CD minimum centered at $205 \mathrm{~nm}$ (Fig. S6). EGCG or EGCG:Zn(II) binding to hIAPP resulted in a decrease in the molar ellipticity indicating the formation of hIAPP aggregates with a mixture of secondary structures (Fig. S6). In the presence of lipid membranes, initially hIAPP adopted a helical structure upon interacting with LUVs as characterized by the two minima observed at 208 and $222 \mathrm{~nm}$ in the CD spectrum. As time progressed the solution generated typical CD spectra of a $\beta$-sheet structure as indicated by a single minimum $\sim 220 \mathrm{~nm}$. In order to quantify the observed structural changes, $\mathrm{CD}$ spectra were obtained at the end point of the reactions in the absence and presence of Zn(II), EGCG (90 min), and 1:1 EGCG:Zn(II) were input for prediction using the BeStSel algorithm.[31]

The results summarized in Table 1 indicate that the initiala-helical structures of hIAPP almost fully converted to $\beta$-sheet amyloid fibrils by showing $\sim 1-4 \%$ of $\alpha$-helical and $\sim 30$ $40 \%$ of $\beta$-sheet. The presence of Zn(II) or EGCG did not significantly alter the secondary structure of hIAPP. The combination of CD and TEM results indicate the formation of $\beta$ sheet structured amyloid fibrils of hIAPP in solution or in a lipid bilayer both in the absence and presence of $\mathrm{Zn}(\mathrm{II})$, while the extent of fibril formation is reduced by the presence of $\mathrm{Zn}$ (II) (Figs. 2A,B and 3A,B, and Table 1). In addition, CD and TEM results indicate the formation of amorphous $\beta$-sheet aggregates in the presence of EGCG or EGCG:Zn(II) as well as the enhanced amyloid-inhibiting capability of EGCG in presence of Zn(II) (Figs. 2, 3 , and S5D,E).

\subsection{Thermodynamic analysis of intermolecular interactions using isothermal titration calorimetry}

To further understand the amyloid inhibiting ability of EGCG, in the absence and presence of $\mathrm{Zn}(\mathrm{II})$, we examined the intermolecular interactions using isothermal titration calorimetry (ITC) experiments (Fig. 4). ITC thermograms showed exothermic binding reactions between hIAPP and EGCG or EGCG:Zn(II) (Fig. 4A and B) indicating intermolecular interactions. ITC analyses revealed that a complex formation was both driven by the negative enthalpy change $(\Delta H)$ and the positive entropy change $(\Delta S)$, and reported the apparent binding stoichiometry ' $n$ ' (the number of hIAPP binding to one EGCG or EGCG:Zn(II)). The values of ' $n$ ' for hIAPP-EGCG and hIAPP-EGCG:Zn(II) binding systems were $~ 0.5$ and $\sim 0.8$, respectively. The dissociation constant $\left(K_{\mathrm{d}}\right)$ of hIAPP for EGCG $(\sim 1.1 \mu \mathrm{M})$ is larger than that for EGCG:Zn(II) mixture $(\sim 0.7 \mu \mathrm{M})$. Thus, the results may implicate that EGCG:Zn(II) complex binds to hIAPP more tightly than EGCG, and more hIAPP is capable of binding to the EGCG:Zn(II) complex as compared to EGCG.

It should be noted that the different reaction environments, such as the ones used in ThT fluorescence or ITC experiments, can produce distinct kinetics of amyloid generation, which 
often makes it difficult to directly compare the kinetics of amyloidogenesis. In the present study, the kinetics of hIAPP aggregation in samples used for ThT-fluorescence experiments and ITC measurements should be different because the sample containers differ in size, surface area of the walls, and the materials used to make the sample cell. In addition, difference in the air-water interface between the microplate used in ThT experiments and ITC cell may also contribute to the difference in the kinetics of aggregation. Based on previous studies, amyloid aggregation has been found to be much slower in an ITC samplecell than that obtained from ThT experiments.[32-34] Because of these reasons, we believe that hIAPP is less likely to be in an aggregated state within the above-mentioned ITC measurement time.

\subsection{Proton NMR shows the formation of large aggregates of hIAPP and EGCG:Zn(II)}

A recent study reported morphologically distinct hIAPP aggregates in presence of EGCG and suggested interactions between hIAPP and EGCG at atomistic resolution.[16] Thus, to further characterize the effects of $\mathrm{Zn}$ (II) on intermolecular interactions and phase states of EGCG as well as on hIAPP amyloid aggregation, we performed a series of proton NMR measurements (Fig. S7, left). NMR spectra showed a broadening of amide-proton resonances of hIAPP in the presence of EGCG (Fig. S7), which is in agreement with a recently reported NMR study.[16] As EGCG aggregates were detected by DLS measurements (Fig. S4) and photography (Fig. S7, right panel), binding of hIAPP to EGCG aggregates (Fig. 4) has been attributed to the source of the observed line broadening. Interestingly, EGCG:Zn(II) significantly reduced the intensities of amide-proton NMR peaks (Fig. S7). In addition, hIAPP mixed with EGCG:Zn(II) yielded a precipitate, whereas a turbid solution of hIAPP with only EGCG was observed (Fig.S7, right panel). Therefore, the observed severe line broadenings are due to interactions between hIAPP and large aggregates of EGCG:Zn(II) complex, as shown by ITC analysis (Fig. 4B).

Based on these experimental results, we suggest that EGCG aggregates serve as inhibitors of amyloid formation, and reason that $\mathrm{Zn}$ (II) binding with EGCG reinforces intermolecular interactions among EGCG and thereby generating larger EGCG aggregates than EGCGalone aggregates without $\mathrm{Zn}$ (II) as observed in DLS measurements (Fig. S4). Indeed, other metal ions such as aluminum have also shown to enhance the anti-amyloidogenic property of EGCG due to complex formation and aggregation.[16] Oligomers of 5,5,6,6tetrachloro-1,1,3,3-tetraethylbenzimidazolyl carbocyanine iodide (JC-1), a fluorescent dye, bound to a-synuclein [35] and oligomers of polyphenolic compounds including EGCG and catechin exhibited higher potency to suppress the fibrillation of amyloid- $\beta$ and insulin than their monomeric forms.[36,37] Taken together, our results so far suggest that aggregated forms of EGCG:Zn(II) are excellent inhibitors of hIAPP aggregation and are more efficient than EGCG-alone. In addition, the observed stabilization of helical structures of hIAPP in LUVs by EGCG or EGCG:Zn(II) (Fig. 3) may be an important factor in the mechanism of amyloid inhibition.

\subsection{MTT assay}

Since we found the EGCG:Zn(II) complex to be efficient in inhibiting amyloid formation of hIAPP, we tested whether it can also counteract the cytotoxicity of hIAPP. We used the MTT 
assay to evaluate the cell viability of freshly-prepared hIAPP or hIAPP aged 24 hours, incubated with either 1 or 5 equivalents of EGCG:Zn(II) for 24 hours (Fig. 5). Similar to published studies, we also observed that monomeric hIAPP was more toxic than the fibrils [7,38]. In contrast, adding 1 or 5 molar equivalents of EGCG:Zn(II) to either $10 \mu \mathrm{M}$ freshlyprepared hIAPP or hIAPP aged 24 hours improved cell viability as compared to hIAPP alone (Fig 5). Interestingly, in the case of $1 \mu \mathrm{M}$ hIAPP, cell viability was improved only for hIAPP incubated with 5 molar equivalents of EGCG:Zn(II) for 24 hours (Figure S8). We did not observe any toxicity for the EGCG:Zn(II) complex alone; in fact, like with EGCG only, we observed an increase in cell viability as compared to cells treated with buffer only (Figs. S8 and S9). This observation is not surprising, as it has also been reported that EGCG increases MTT-related viability.[17] Treating cells with $\mathrm{Zn}$ in the presence and absence of hIAPP reduced the viability, suggesting the toxic nature of free $\mathrm{Zn}$ (II). Cell toxicity measurements were also performed with hIAPP with either EGCG or Zn(II) separately (Fig. S9). Treating hIAPP with EGCG, increased cell viability compared to EGCG:Zn(II). Additionally, EGCG:Zn(II) was found to be less toxic than $\mathrm{Zn}$ (II) alone, indicating the ability of EGCG to neutralize it's toxicity (Fig S9). This shows the enhancement of hIAPP toxicity by Zn(II) and thus the cell viability enhancement of EGCG. While the EGCG:Zn(II) complex is not as efficient as EGCG in promoting cell viability, it does show the ability of complex formation on rescuing $\mathrm{Zn}(\mathrm{II})$ toxicity.

Previous studies have reported the toxic nature of the EGCG:Zn(II) complex at higher concentrations on a different cell line than the ones used in this study in the absence of peptide.[29,39] Interestingly, Sun et al. [29] showed that the cell permeability is enhanced by the presence of free Zn(II). However, EGCG complexed with Zn(II) alleviates the toxic effect of the metal ion. While $\mathrm{Zn}$ (II) can induce off-pathway toxic aggregates [40], adding EGCG counteracts its toxic effect and the EGCG:Zn(II) complex exhibits protective effect on the cells as indicated by our results.

\section{Conclusions}

EGCG has been shown to be an efficient inhibitor of amyloid aggregation by a variety of amyloidogenic peptides and proteins, and has also been shown to remodel amyloid fibers. [41-52] Although many small molecules can inhibit amyloid aggregation in solution, it is important to evaluate the efficiency of such compounds in the presence of membranes, which could be directly related to their ability to suppress cell toxicity.[26,53-55] In this study, we have successfully demonstrated the ability of EGCG to suppress the amyloid fibril formation of hIAPP both in solution and in the presence of membranes by using a combination of ThT fluorescence, TEM and CD experiments. In addition, the reported results demonstrate that the presence of Zn(II) reduced the amount of EGCG required to fully inhibit hIAPP's fibril formation. These results are further confirmed by the cell toxicity measurements that show the ability of EGCG:Zn(II) complex to significantly suppress hIAPP's toxicity. In fact, EGCG suppress zinc's toxicity to cells as observed from the significant increase in cell viability in the presence of EGCG:Zn(II) complex. We believe that the results reported in this study provide insightful information for the future development of therapeutic small molecules targeting amyloid diseases. 
While the EGCG:Zn(II) complex has been shown to be efficient in suppressing hIAPP's amyloid fibril formation, it would be useful to investigate the structural effects of zinc on EGCG and the factors that enhances the efficacy of EGCG:Zn(II) complex. Our NMR experiments showed that the presence of zinc enhanced the aggregation of EGCG-hIAPP to result in sample precipitation. A systematic analysis of the aggregated and/or precipitated samples by magic angle spinning solid-state NMR experiments would provide insights into EGCG-Zn(II) and EGCG:Zn(II)hIAPP interactions. It is remarkable that EGCG:Zn(II) complex stabilizes helical oligomeric hIAPP intermediates in a membrane environment within the elongated lag-time as revealed by $\mathrm{CD}$ experiments. High-resolution structures of such intermediates can be determined by NMR experiments, although the intermediate structures may have to be stabilized by cooling (or freezing) the sample for MAS solid-state NMR experiments. Structures of these intermediates would be useful to better understand the aggregation pathways as well as the mechanism of amyloid inhibition by EGCG or EGCG:Zn(II).

\section{Supplementary Material}

Refer to Web version on PubMed Central for supplementary material.

\section{Acknowledgements:}

This study was supported by NIH (AG048934 to A.R.) and a Grant-in-Aid for Young Scientists (B) (15K18518 and 25870407) (to Y.-H.L.)

\section{References}

1. Westermark P, Andersson A, Westermark GT: Islet amyloid polypeptide, islet amyloid, and diabetes mellitus. Physiol Rev 2011, 91:795-826. [PubMed: 21742788]

2. Kahn SE, D’Alessio DA, Schwartz MW, Fujimoto WY, Ensinck JW, Taborsky GJ, Porte D: Evidence of cosecretion of islet amyloid polypeptide and insulin by beta-cells. Diabetes 1990, 39:634-638. [PubMed: 2185112]

3. Marzban L, Park K, Verchere CB: Islet amyloid polypeptide and type 2 diabetes. Exp Gerontol 2003, 38:347351. [PubMed: 12670620]

4. Brender JR, Salamekh S, Ramamoorthy A: Membrane disruption and early events in the aggregation of the diabetes related peptide IAPP from a molecular perspective. Acc Chem Res 2012, 45:454462. [PubMed: 21942864]

5. Sciacca MF, Brender JR, Lee DK, Ramamoorthy A: Phosphatidylethanolamine enhances amyloid fiberdependent membrane fragmentation. Biochemistry 2012, 51:7676-7684. [PubMed: 22970795]

6. Nanga RP, Brender JR, Vivekanandan S, Ramamoorthy A: Structure and membrane orientation of IAPP in its natively amidated form at physiological $\mathrm{pH}$ in a membrane environment. Biochim Biophys Acta 2011, 1808:2337-2342. [PubMed: 21723249]

7. Abedini A, Plesner A, Cao P, Ridgway Z, Zhang J, Tu LH, Middleton CT, Chao B, Sartori DJ, Meng F, et al.: Time-resolved studies define the nature of toxic IAPP intermediates, providing insight for antiamyloidosis therapeutics. Elife 2016, 5.

8. Birol M, Kumar S, Rhoades E, Miranker AD: Conformational switching within dynamic oligomers underpins toxic gain-of-function by diabetes-associated amyloid. Nat Commun 2018, 9:1312. [PubMed: 29615609]

9. Doig AJ, Derreumaux P: Inhibition of protein aggregation and amyloid formation by small molecules. Curr Opin Struct Biol 2015, 30:50-56. [PubMed: 25559306] 
10. Sahoo BR, Genjo T, Bekier M, Cox SJ, Stoddard AK, Ivanova M, Yasuhara K, Fierke CA, Wang Y, Ramamoorthy A: Alzheimer's amyloid-beta intermediates generated using polymer-nanodiscs. Chem Commun (Camb) 2018.

11. Velander P, Wu L, Henderson F, Zhang S, Bevan DR, Xu B: Natural product-based amyloid inhibitors. Biochem Pharmacol 2017, 139:40-55. [PubMed: 28390938]

12. Popovych N, Brender JR, Soong R, Vivekanandan S, Hartman K, Basrur V, Macdonald PM, Ramamoorthy A: Site specific interaction of the polyphenol EGCG with the SEVI amyloid precursor peptide PAP(248286). J Phys Chem B 2012, 116:3650-3658. [PubMed: 22360607]

13. Hora M, Carballo-Pacheco M, Weber B, Morris VK, Wittkopf A, Buchner J, Strodel B, Reif B: Epigallocatechin3-gallate preferentially induces aggregation of amyloidogenic immunoglobulin light chains. Sci Rep 2017, 7:41515. [PubMed: 28128355]

14. Lorenzen N, Nielsen SB, Yoshimura Y, Vad BS, Andersen CB, Betzer C, Kaspersen JD, Christiansen G, Pedersen JS, Jensen PH, et al.: How epigallocatechin gallate can inhibit alphasynuclein oligomer toxicity in vitro. J Biol Chem 2014, 289:21299-21310. [PubMed: 24907278]

15. Andrich K, Bieschke J: The Effect of (-)-Epigallo-catechin-(3)-gallate on Amyloidogenic Proteins Suggests a Common Mechanism. Adv Exp Med Biol 2015, 863:139-161. [PubMed: 26092630]

16. Franko A, Rodriguez Camargo DC, Boddrich A, Garg D, Rodriguez Camargo A, Rathkolb B, Janik D, Aichler M, Feuchtinger A, Neff F, et al.: Epigallocatechin gallate (EGCG) reduces the intensity of pancreatic amyloid fibrils in human islet amyloid polypeptide (hIAPP) transgenic mice. Sci Rep 2018, 8:1116. [PubMed: 29348618]

17. Meng F, Abedini A, Plesner A, Verchere CB, Raleigh DP: The flavanol (-)-epigallocatechin 3gallate inhibits amyloid formation by islet amyloid polypeptide, disaggregates amyloid fibrils, and protects cultured cells against IAPP-induced toxicity. Biochemistry 2010, 49:8127-8133. [PubMed: 20707388]

18. Bulic B, Pickhardt M, Mandelkow E: Progress and developments in tau aggregation inhibitors for Alzheimer disease. J Med Chem 2013, 56:4135-4155. [PubMed: 23484434]

19. Guo C, Ma L, Zhao Y, Peng A, Cheng B, Zhou Q, Zheng L, Huang K: Inhibitory effects of magnolol and honokiol on human calcitonin aggregation. Sci Rep 2015, 5:13556. [PubMed: 26324190]

20. Ehrnhoefer DE, Bieschke J, Boeddrich A, Herbst M, Masino L, Lurz R, Engemann S, Pastore A, Wanker EE: EGCG redirects amyloidogenic polypeptides into unstructured, off-pathway oligomers. Nat Struct Mol Biol 2008, 15:558-566. [PubMed: 18511942]

21. Bieschke J, Russ J, Friedrich RP, Ehrnhoefer DE, Wobst H, Neugebauer K, Wanker EE: EGCG remodels mature alpha-synuclein and amyloid-beta fibrils and reduces cellular toxicity. Proc Natl Acad Sci U S A 2010, 107:7710-7715. [PubMed: 20385841]

22. Foster MC, Leapman RD, Li MX, Atwater I: Elemental composition of secretory granules in pancreatic islets of Langerhans. Biophys J 1993, 64:525-532. [PubMed: 8457676]

23. Salamekh S, Brender JR, Hyung SJ, Nanga RP, Vivekanandan S, Ruotolo BT, Ramamoorthy A: A two-site mechanism for the inhibition of IAPP amyloidogenesis by zinc. J Mol Biol 2011, 410:294-306. [PubMed: 21616080]

24. Brender JR, Hartman K, Nanga RP, Popovych N, de la Salud Bea R, Vivekanandan S, Marsh EN, Ramamoorthy A: Role of zinc in human islet amyloid polypeptide aggregation. J Am Chem Soc 2010, 132:8973-8983. [PubMed: 20536124]

25. Brender JR, Krishnamoorthy J, Messina GM, Deb A, Vivekanandan S, La Rosa C, Penner-Hahn JE, Ramamoorthy A: Zinc stabilization of prefibrillar oligomers of human islet amyloid polypeptide. Chem Commun (Camb) 2013, 49:3339-3341. [PubMed: 23505632]

26. Pithadia AS, Bhunia A, Sribalan R, Padmini V, Fierke CA, Ramamoorthy A: Influence of a curcumin derivative on hIAPP aggregation in the absence and presence of lipid membranes. Chem Commun (Camb) 2016, 52:942-945. [PubMed: 26587568]

27. Terakawa MS, Yagi H, Adachi M, Lee YH, Goto Y: Small liposomes accelerate the fibrillation of amyloid beta (1-40). J Biol Chem 2015, 290:815-826. [PubMed: 25406316]

28. Nielsen L, Khurana R, Coats A, Frokjaer S, Brange J, Vyas S, Uversky VN, Fink AL: Effect of environmental factors on the kinetics of insulin fibril formation: elucidation of the molecular mechanism. Biochemistry 2001, 40:6036-6046. [PubMed: 11352739] 
29. Sun SL, He GQ, Yu HN, Yang JG, Borthakur D, Zhang LC, Shen SR, Das UN: Free Zn(2+) enhances inhibitory effects of EGCG on the growth of PC-3 cells. Mol Nutr Food Res 2008, 52:465-471. [PubMed: 18324707]

30. Suzuki Y, Brender JR, Hartman K, Ramamoorthy A, Marsh EN: Alternative pathways of human islet amyloid polypeptide aggregation distinguished by (19)f nuclear magnetic resonance-detected kinetics of monomer consumption. Biochemistry 2012, 51:8154-8162. [PubMed: 22998665]

31. Micsonai A, Wien F, Kernya L, Lee YH, Goto Y, Refregiers M, Kardos J: Accurate secondary structure prediction and fold recognition for circular dichroism spectroscopy. Proc Natl Acad Sci U S A 2015, 112:E3095-3103. [PubMed: 26038575]

32. Ikenoue T, Lee YH, Kardos J, Saiki M, Yagi H, Kawata Y, Goto Y: Cold denaturation of asynuclein amyloid fibrils. Angew Chem Int Ed Engl 2014, 53:7799-7804.

33. Ikenoue T, Lee YH, Kardos J, Yagi H, Ikegami T, Naiki H, Goto Y: Heat of supersaturation-limited amyloid burst directly monitored by isothermal titration calorimetry. Proc Natl Acad Sci U S A 2014, 111:66546659. [PubMed: 24753579]

34. Kinoshita M, Lin Y, Dai I, Okumura M, Markova N, Ladbury JE, Sterpone F, Lee YH: Energy landscape of polymorphic amyloid generation of $\beta 2$-microglobulin revealed by calorimetry. Chem Commun (Camb) 2018, 54:7995-7998. [PubMed: 29967909]

35. Lee JH, Lee IH, Choe YJ, Kang S, Kim HY, Gai WP, Hahn JS, Paik SR: Real-time analysis of amyloid fibril formation of alpha-synuclein using a fibrillation-state-specific fluorescent probe of JC-1. Biochem J 2009, 418:311-323. [PubMed: 19007333]

36. Nie RZ, Zhu W, Peng JM, Ge ZZ, Li CM: A-type dimeric epigallocatechin-3-gallate (EGCG) is a more potent inhibitor against the formation of insulin amyloid fibril than EGCG monomer. Biochimie 2016, 125:204-212. [PubMed: 27079519]

37. Hayden EY, Yamin G, Beroukhim S, Chen B, Kibalchenko M, Jiang L, Ho L, Wang J, Pasinetti GM, Teplow DB: Inhibiting amyloid $\beta$-protein assembly: Size-activity relationships among grape seed-derived polyphenols. J Neurochem 2015, 135:416-430. [PubMed: 26228682]

38. Sciacca MF, Kotler SA, Brender JR, Chen J, Lee DK, Ramamoorthy A: Two-step mechanism of membrane disruption by Abeta through membrane fragmentation and pore formation. Biophys $\mathbf{J}$ 2012, 103:702710. [PubMed: 22947931]

39. Yang J, Yu H, Sun S, Zhang L, Das UN, Ruan H, He G, Shen S: Mechanism of free Zn(2+) enhancing inhibitory effects of EGCG on the growth of PC-3 cells: interactions with mitochondria. Biol Trace Elem Res 2009, 131:298-310. [PubMed: 19326061]

40. Lee MC, Yu WC, Shih YH, Chen CY, Guo ZH, Huang SJ, Chan JCC, Chen YR: Zinc ion rapidly induces toxic, off-pathway amyloid- $\beta$ oligomers distinct from amyloid- $\beta$ derived diffusible ligands in Alzheimer's disease. Sci Rep 2018, 8:4772. [PubMed: 29555950]

41. Palhano FL, Lee J, Grimster NP, Kelly JW: Toward the molecular mechanism(s) by which EGCG treatment remodels mature amyloid fibrils. J Am Chem Soc 2013, 135:7503-7510. [PubMed: 23611538]

42. Lopez del Amo JM, Fink U, Dasari M, Grelle G, Wanker EE, Bieschke J, Reif B: Structural properties of EGCG-induced, nontoxic Alzheimer's disease A $\beta$ oligomers. J Mol Biol 2012, 421:517-524. [PubMed: 22300765]

43. Hyung SJ, DeToma AS, Brender JR, Lee S, Vivekanandan S, Kochi A, Choi JS, Ramamoorthy A, Ruotolo BT, Lim MH: Insights into antiamyloidogenic properties of the green tea extract (-)epigallocatechin-3gallate toward metal-associated amyloid- $\beta$ species. Proc Natl Acad Sci U S A 2013, 110:3743-3748. [PubMed: 23426629]

44. Huang R, Vivekanandan S, Brender JR, Abe Y, Naito A, Ramamoorthy A: NMR characterization of monomeric and oligomeric conformations of human calcitonin and its interaction with EGCG. J Mol Biol 2012, 416:108-120. [PubMed: 22200484]

45. Fusco G, Sanz-Hernandez M, Ruggeri FS, Vendruscolo M, Dobson CM, De Simone A: Molecular determinants of the interaction of EGCG with ordered and disordered proteins. Biopolymers 2018:e23117. [PubMed: 29603125]

46. Jha NN, Kumar R, Panigrahi R, Navalkar A, Ghosh D, Sahay S, Mondal M, Kumar A, Maji SK: Comparison of a-Synuclein Fibril Inhibition by Four Different Amyloid Inhibitors. ACS Chem Neurosci 2017, 8:27222733. [PubMed: 28872299] 
47. Ahmed R, Melacini G: A solution NMR toolset to probe the molecular mechanisms of amyloid inhibitors. Chem Commun (Camb) 2018, 54:4644-4652. [PubMed: 29658548]

48. Tu LH, Young LM, Wong AG, Ashcroft AE, Radford SE, Raleigh DP: Mutational analysis of the ability of resveratrol to inhibit amyloid formation by islet amyloid polypeptide: critical evaluation of the importance of aromatic-inhibitor and histidine-inhibitor interactions. Biochemistry 2015, 54:666-676. [PubMed: 25531836]

49. Xu ZX, Ma GL, Zhang Q, Chen CH, He YM, Xu LH, Zhou GR, Li ZH, Yang HJ, Zhou P: Inhibitory Mechanism of Epigallocatechin Gallate on Fibrillation and Aggregation of Amidated Human Islet Amyloid Polypeptide. Chemphyschem 2017, 18:1611-1619. [PubMed: 28297133]

50. Mo Y, Lei J, Sun Y, Zhang Q, Wei G: Conformational Ensemble of hIAPP Dimer: Insight into the Molecular Mechanism by which a Green Tea Extract inhibits hIAPP Aggregation. Sci Rep 2016, 6:33076. [PubMed: 27620620]

51. Wang J, Yamamoto T, Bai J, Cox SJ, Korshavn KJ, Monette M, Ramamoorthy A: Real-time monitoring of the aggregation of Alzheimer's amyloid- $\beta$ via. Chem Commun (Camb) 2018, 54:2000-2003. [PubMed: 29411841]

52. Pithadia A, Brender JR, Fierke CA, Ramamoorthy A: Inhibition of IAPP Aggregation and Toxicity by Natural Products and Derivatives. J Diabetes Res 2016, 2016:2046327. [PubMed: 26649317]

53. Sahoo BR, Genjo T, Cox SJ, Stoddard AK, Anantharamaiah GM, Fierke C, Ramamoorthy A: Nanodisc-Forming Scaffold Protein Promoted Retardation of Amyloid-Beta Aggregation. J Mol Biol 2018, 430:4230-4244. [PubMed: 30170005]

54. Malishev R, Shaham-Niv S, Nandi S, Kolusheva S, Gazit E, Jelinek R: Bacoside-A, an Indian TraditionalMedicine Substance, Inhibits $\beta$-Amyloid Cytotoxicity, Fibrillation, and Membrane Interactions. ACS Chem Neurosci 2017, 8:884-891. [PubMed: 28094495]

55. Engel MF, vandenAkker CC, Schleeger M, Velikov KP, Koenderink GH, Bonn M: The polyphenol EGCG inhibits amyloid formation less efficiently at phospholipid interfaces than in bulk solution. J Am Chem Soc 2012, 134:14781-14788. [PubMed: 22889183] 


\section{Highlights}

- $\quad$ EGCG:Zn(II) inhibits amyloid aggregation in solution as well as in the presence of membrane.

- $\quad$ EGCG:Zn(II) complex efficiently suppresses human-IAPP's toxicity to pancreatic $\beta$-cells.

- $\quad$ EGCG suppresses Zinc's toxicity to pancreatic $\beta$-cells.

- Human-IAPP forms a helical structure in presence of EGCG:Zn(II) in membrane. 
A

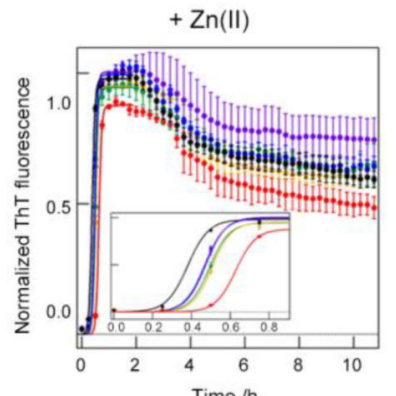

D

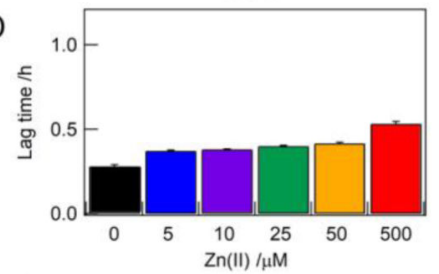

E

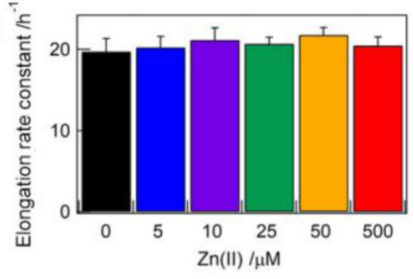

$\mathrm{F}$

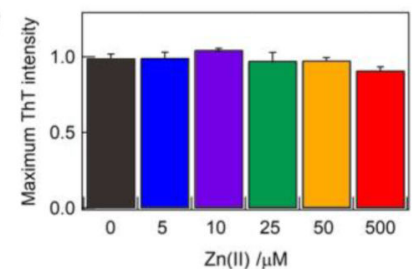

B
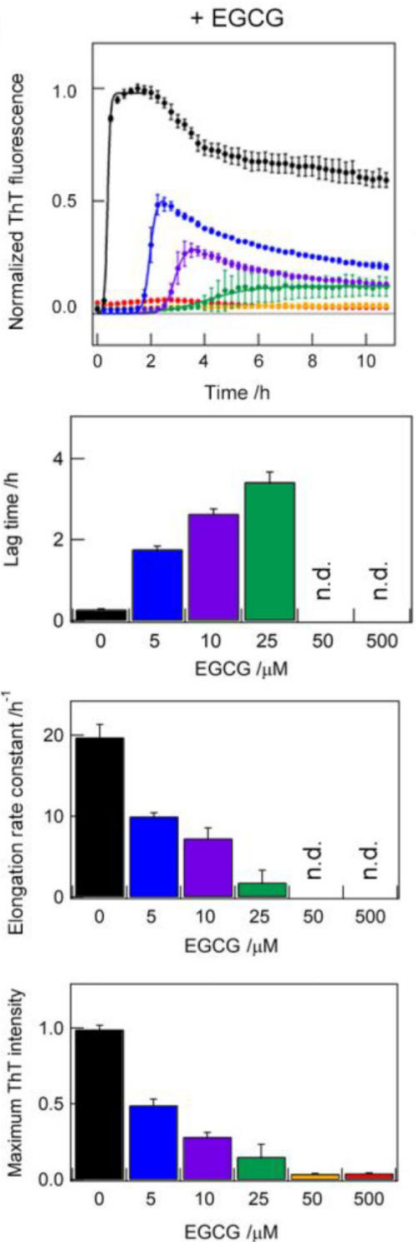

C
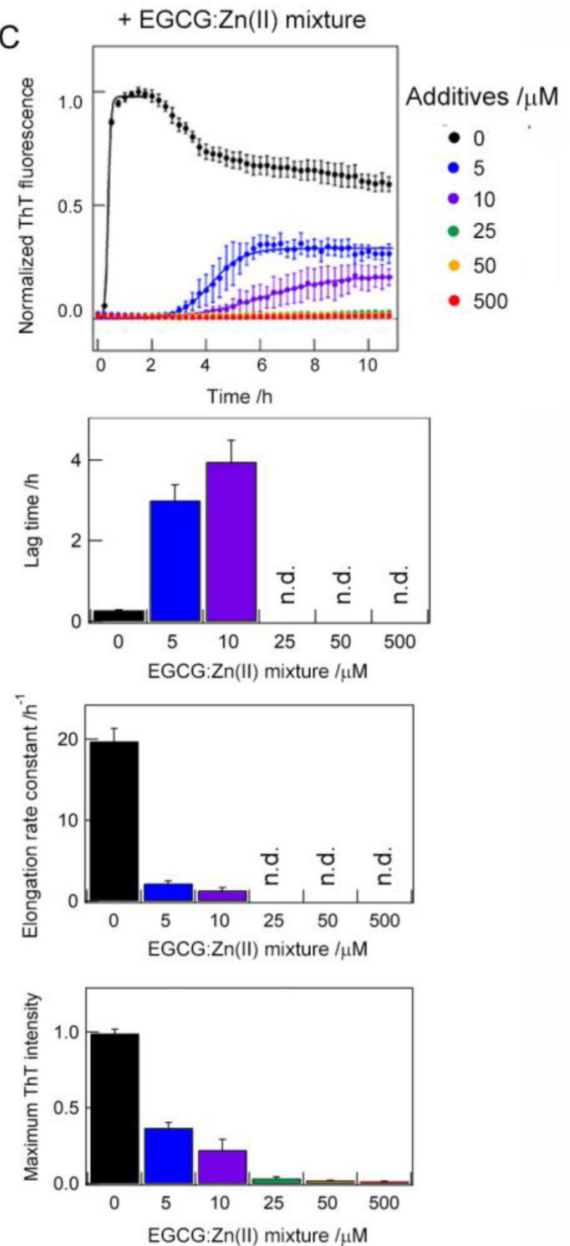

Figure 1. Inhibition of hIAPP amyloid formation in a lipid bilayer.

(A-C) ThT fluorescence experiments showing the suppression of amyloid aggregation of hIAPP by Zn(II) (A), EGCG (B), EGCG:Zn(II) mixture and (C) in the presence of 7:3 POPC:POPG LUVs. Lag time (D), elongation rate constant (E) and maximum ThT intensity (F) in the presence of $\mathrm{Zn}$ (II) (left), EGCG (middle) and EGCG:Zn(II) mixture (right). hIAPP of $5 \mu \mathrm{M}$ was incubated at $37^{\circ} \mathrm{C}$ and $\mathrm{pH}$ 7.4. Average values of three independent measurements are shown with error bars representing the standard deviation. The inset in Fig. 1A is shown to indicate the differences in the rapid kinetics of hIAPP fibrillation with and without $\mathrm{Zn}(\mathrm{II})$. Solid lines in A-D are the best-fit of experimental data points. "n.d." denotes the concentration at which the best fit was not obtained due to very slow aggregation (i.e., very low ThT intensity). 
A

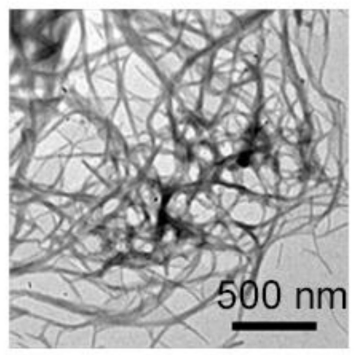

D

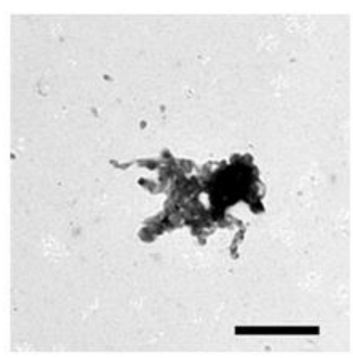

B

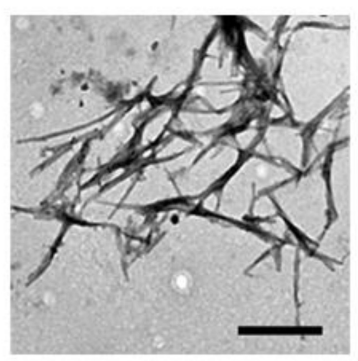

$E$

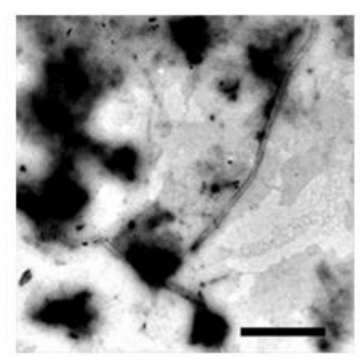

C

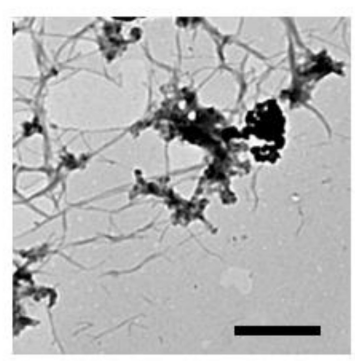

$\mathrm{F}$

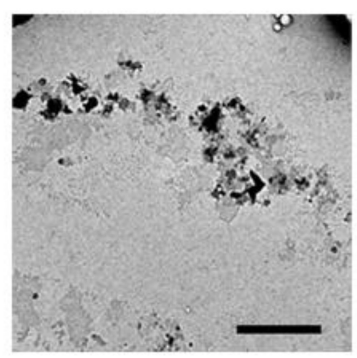

Figure 2. TEM images of hIAPP aggregates.

Mature amyloid fibrils of hIAPP of $5 \mu \mathrm{M}$ incubated for 12 hours with 7:3 POPC:POPG LUVs in the absence of additives (A) and in the presence of $500 \mu \mathrm{M} \mathrm{Zn}$ (II) (B), $5 \mu \mathrm{M}$ EGCG (C), $500 \mu \mathrm{M}$ EGCG (D), $5 \mu \mathrm{M}$ EGCG:Zn(II) mixture (1:1 molar ratio) (E), and 500 $\mu \mathrm{M}$ EGCG:Zn(II) mixture (1:1 molar ratio) (F). The black scale bars represent $500 \mathrm{~nm}$. 



C
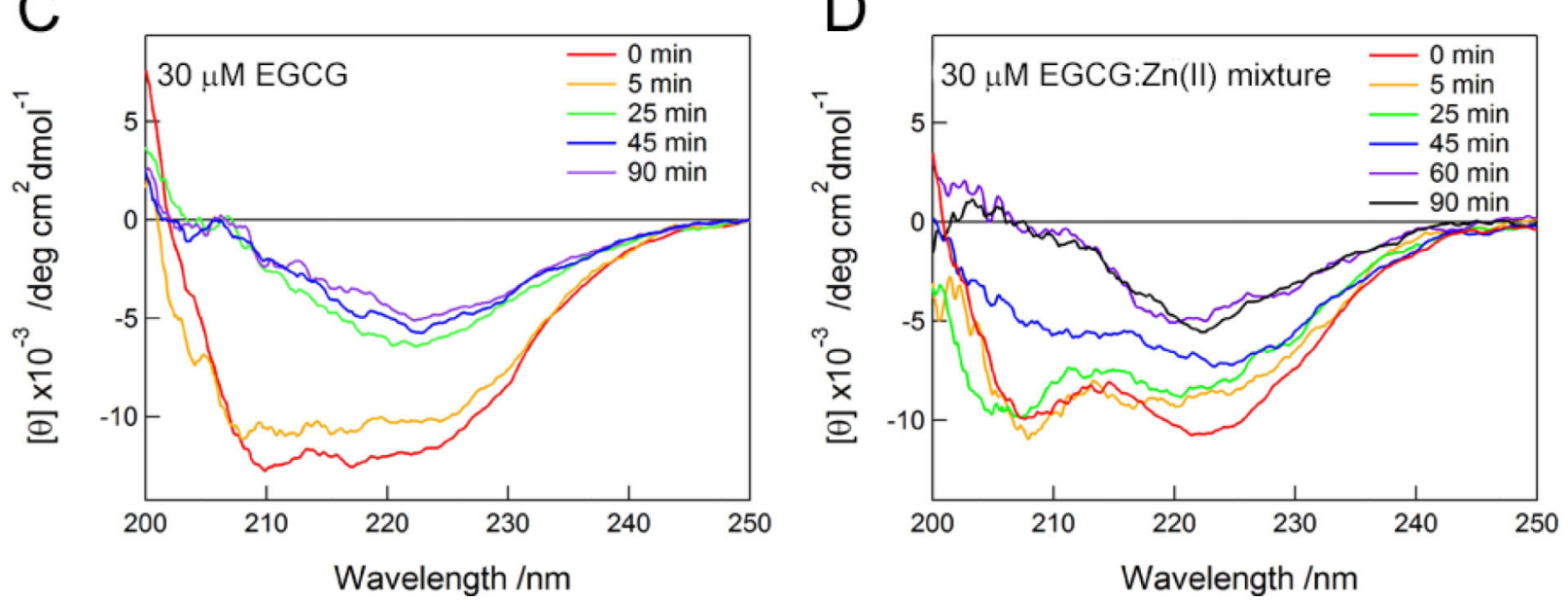

Figure 3. Real time CD spectra of hIAPP.

Far-UV CD spectra of hIAPP at $15 \mu \mathrm{M}$ in 7:3 POPC:POPG LUVs (A) and in the presence of $30 \mu \mathrm{M} Z \mathrm{Zn}$ (II) (B), $30 \mu \mathrm{M}$ EGCG (C), and $30 \mu \mathrm{M}$ of 1:1 molar ratio of EGCG:Zn(II) mixture (D). The formation and stabilization of a helical structure of hIAPP in presence of EGCG:Zn(II) complex for a long duration is worth mentioning and could be important in enabling high-resolution structural studies of the intermediate. 
A

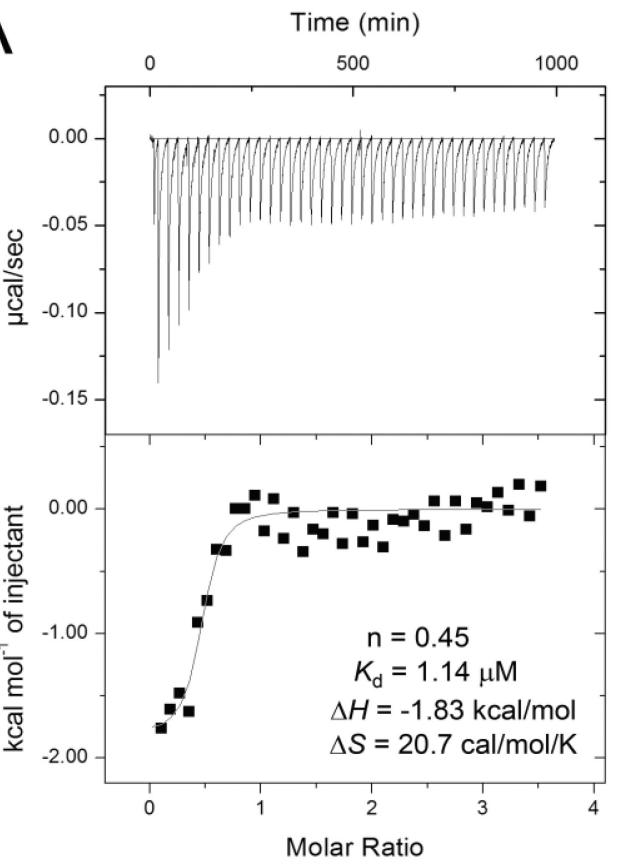

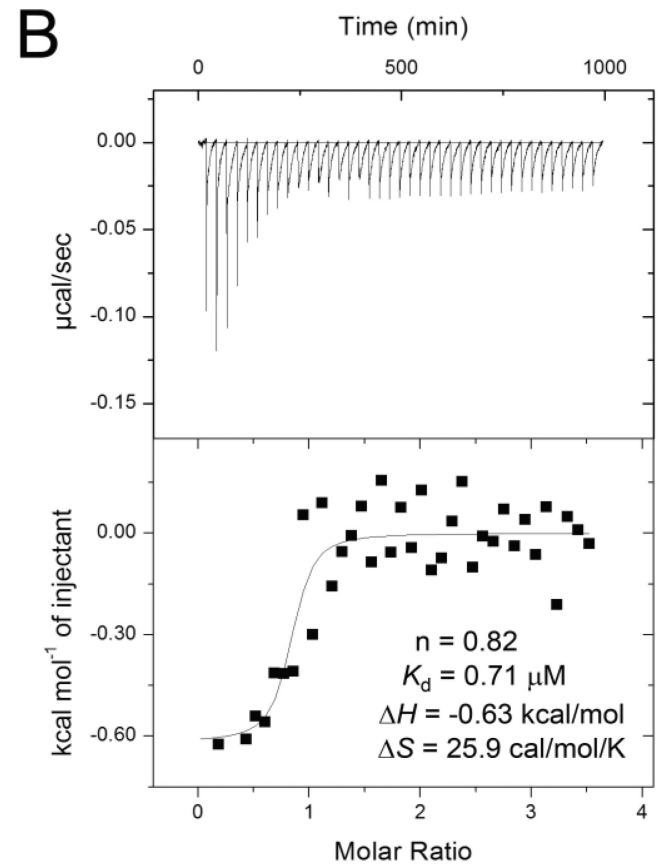

Figure 4. ITC measurements for the interaction between hIAPP and EGCG or EGCG:Zn(II). (A and B) ITC thermograms (top) and binding isotherms (bottom) for the titration of $1 \mathrm{mM}$ EGCG (A) and EGCG:Zn(II) (B) into $60 \mu \mathrm{M}$ hIAPP. 


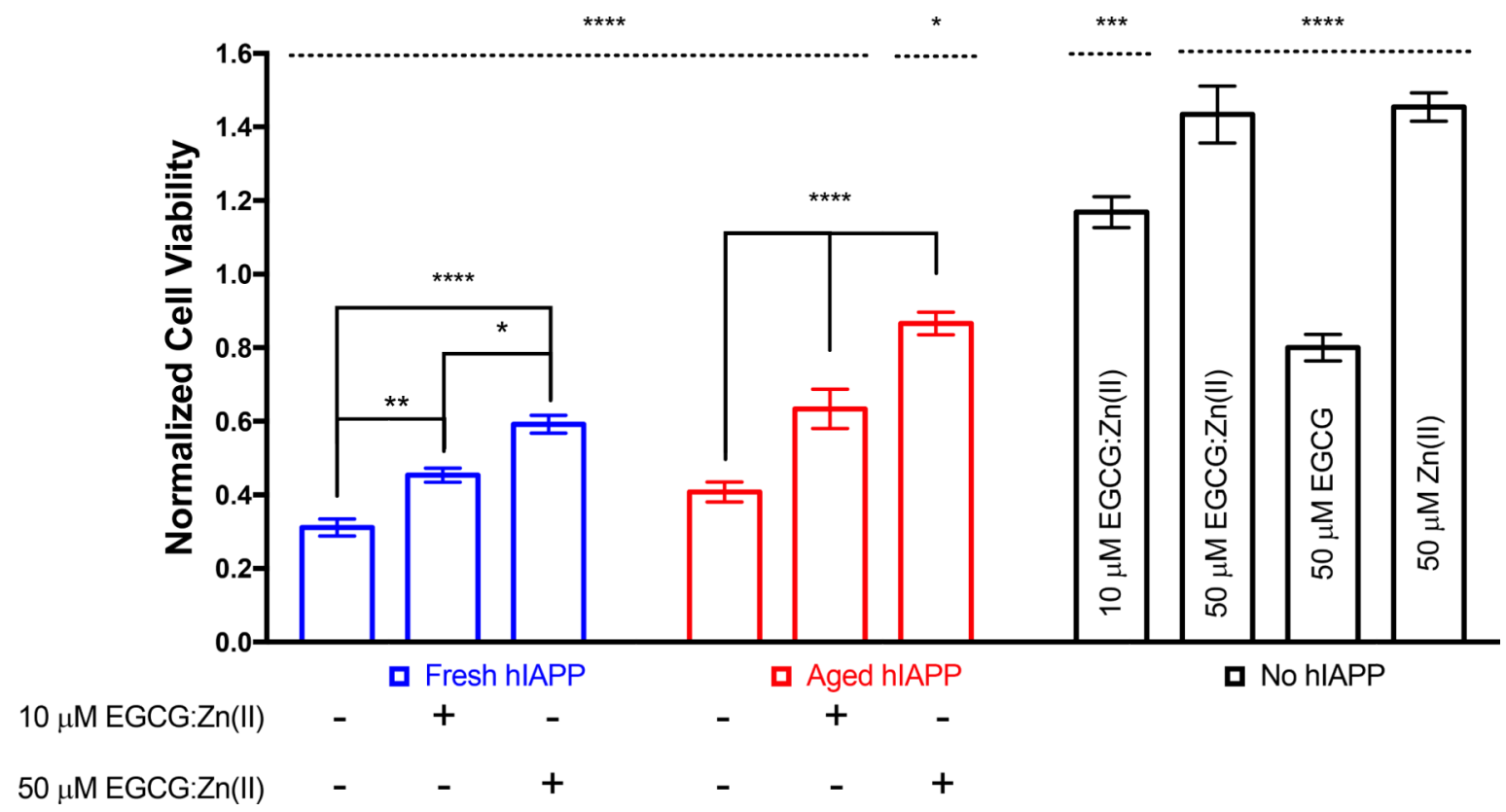

Figure 5. EGCG:Zn(II) complex rescues the cell viability of RIN-5F cells.

EGCG:Zn(II) (1 or 5 molar equivalents) increased cellular viability for both fresh and 24 hours aged $10 \mu \mathrm{M}$ hIAPP measured by MTT assays. Samples were normalized with MTT absorbance of buffer and 1\% SDS as negative and positive controls, respectively. Ordinary one-way ANOVA tests with Tukey's multiple comparisons were performed in respect to buffer treated cells (dotted line) and in respect to individual samples (solid line). ****pvalue $<0.0001, * * * \mathrm{p}$-value $<0.0002, * * \mathrm{p}<0.01$ and $* \mathrm{p}<0.05$ indicate levels of significant differences. Data is shown as mean $+/-\mathrm{SD}$ with $\mathrm{N}=5$ replicates. $(+)$ Indicates addition of sample to cells, (-) means sample was not added to cells. 
Table 1.

Secondary structure of hIAPP aggregates determined from CD experiments.

\begin{tabular}{ccc}
\hline Samples & $\boldsymbol{a}$-Helix (\%) & $\boldsymbol{\beta}$-Sheet (\%) \\
\hline hIAPP alone & 1.8 & 43.6 \\
hIAPP + 30 $\mu$ M Zn(II) & 0.8 & 31.2 \\
hIAPP + 30 $\mu$ M EGCG & 3.8 & 30.1 \\
hIAPP + 30 $\mu$ M EGCG:Zn(II) mixture & 0.5 & 34.9 \\
\hline
\end{tabular}

$a$-helical and $\beta$-sheet conformations of hIAPP were determined from CD spectra obtained at the end of the reactions in the absence (after 45 min of incubation) and presence of Zn(II) (after 45 min of incubation), EGCG ( after 90 min of incubation ), and 1:1 EGCG:Zn(II) ( after 90 min of incubation) using the BeStSel algorithm. 